\title{
Neurosurgeons in 2020: the impact of gender on neurosurgical training, family planning, and workplace culture
}

\author{
Jasmine A. Thum, MD, MSE, ${ }^{1}$ Diana Chang, MA, MS, ${ }^{2}$ Nalini Tata, MPhil, ${ }^{3}$ and \\ Linda M. Liau, MD, PhD, MBA ${ }^{1}$
}

1Department of Neurosurgery, University of California, Los Angeles; '2Department of Neurosurgery, UCSF School of Medicine, University of California, San Francisco, California; and ${ }^{3}$ Department of Neurosurgery, Northwestern Feinberg School of Medicine, Chicago, Illinois

OBJECTIVE In 2008, a Women in Neurosurgery Committee white paper called for increased women applicants and decreased women's attrition in neurosurgery. However, contributing factors (work-life balance, lack of female leadership, workplace gender inequality) have not been well characterized; therefore, specific actions cannot be implemented to improve these professional hurdles. This study provides an update on the experiences of neurosurgeons in 2020 with these historical challenges.

METHODS An anonymous online survey was sent to all Accreditation Council for Graduate Medical Education (ACGME)-accredited US neurosurgical programs, examining demographics and experiences with mentorship, family life, fertility, and workplace conduct.

RESULTS A total of 115 respondents (64 men, 51 women; age range 25-67 years) had trained at 49 different US residencies. Mentorship rates were very high among men and women in medical school and residency. However, women were significantly more likely than men to have a female mentor in residency. During residency, $33 \%$ of women versus $44 \%$ of men had children, and significantly fewer women interested in having a child were able to do so in residency, compared to men. Significantly more women than men had a child only during a nonclinical year $(56.3 \%$ vs $19.0 \%$, respectively). Thirty-nine percent of women and $25 \%$ of men reported difficulty conceiving. The major difficulty for men was stress, whereas women reported the physical challenges of pregnancy itself (workplace teratogens, morning sickness, etc.). Failed birth rates peaked during residency $(0.33)$ versus those before $(0.00)$ and after residency $(0.25)$. Women (80\%) experience microaggressions in the workplace significantly more than men $(36 \% ; p<0.001)$. Ninety-five percent of macro-/microaggressions toward female neurosurgeons were about their gender, compared to $9 \%$ of those toward men $(p<0.001)$. The most common overall perpetrators were senior male residents and attendings, followed by male patients (against women) and female nurses or midlevel providers (against men).

CONCLUSIONS Accurate depictions of neurosurgery experiences and open discussions of the potential impacts of gender may allow for 1) decreased attrition due to more accurate expectations and 2) improved characterization of gender differences in neurosurgery so the profession can work to address gender inequality.

https://thejns.org/doi/abs/10.3171/2020.12.FOCUS20965

KEYWORDS women in neurosurgery; mentorship; family planning; workplace culture; gender disparities; diversity

I N 2008, a seminal white paper authored by the leadership of Women in Neurosurgery and commissioned by the American Association of Neurological Surgeons provided a comprehensive analysis of the recruitment and retainment of women in the field. The paper remarked that despite the fact that more women than men were enrolling in medical school, women were significantly under- represented in neurosurgery, composing only $5.9 \%$ of practicing neurosurgeons at the time. The white paper laid out two major goals, advocating that women make up 1) $20 \%$ of each class entering residency by the year 2012 and 2) $20 \%$ of all neurosurgery faculty by the year $2020 .{ }^{1}$ In 2020, while we made progress, the field has yet to achieve either of these goals. 
Around the time the white paper was written, neurosurgery had the lowest percentage of women of any accredited medical specialty (11.15\%, 2007-2008 academic year), and female gender was a negative predictive factor for matching into neurosurgical residency when controlling for Step scores and Alpha Omega Alpha Honor Medical Society (AOA) status. ${ }^{2}$ A decade later, in 2017-2018, the percentage of women neurosurgery trainees had risen by $6.67 \%$ to $17.82 \%$ of female residents overall; neurosurgery ranked lowest in terms of the percentage of female trainees of any medical specialty, save for only orthopedic surgery ( $15.40 \%$ women). ${ }^{3}$ While these data reveal promising progress toward the goal of $20 \%$, albeit a few years behind schedule, the professional progression of women in neurosurgery beyond residency remains a concern, as there are still significant gender disparities in attrition rates and in high-level academic appointments.

Women have historically had a higher rate of attrition from neurosurgery than their male counterparts. A study of female neurosurgery residents between 1990 and 1999 found that $40 \%$ of those women did not become board certified, a rate twice as high as for men. ${ }^{4}$ An updated analysis of female neurosurgery residents between 2000 and 2009 found that female attrition decreased to $17 \%$ but was still significantly higher than the male attrition rate of $5.3 \%$ over that same period. ${ }^{5}$

The gender gap in neurosurgery widens with increasing academic rank. A 2016 study found that while women constituted $16.3 \%$ of neurosurgery residents, they represented only $6.1 \%$ of board-certified neurosurgeons and only $4 \%$ of full professors of neurosurgery at the time. ${ }^{6}$ Similarly, a 2019 study highlighted a significant underrepresentation of women in academic neurosurgery. While the proportion of male and female residents entering academic neurosurgery after residency is roughly equal, there is a steep decline in the number of women at higher levels within academia. As of 2019, only 5.84\% of full professors of neurosurgery were women, ${ }^{7}$ and in 2020 , there were only 4 female chairs of neurosurgery across the country.

A number of factors have been suggested to explain these ongoing disparities. Broadly speaking, these have been characterized as lifestyle concerns (i.e., family planning), discrimination, traditional career structures, lack of mentors, and personal perception (satisfaction, goals). ${ }^{8}$ In order to provide an update to the white paper goals and assess the impact of some of these variables in the current context of neurosurgical training, we conducted a survey of resident and attending attitudes and experiences in neurosurgery in 2020.

\section{Methods}

We obtained exemption from the University of California, Los Angeles IRB for this survey study. Snowball sampling was achieved by distributing the survey to residency program coordinators to send to neurosurgeons in training and in practice in their programs. The survey was open for 2 weeks with two reminder emails to increase participation.

The anonymous survey consisted of four components:
1) demographic information including age, sex, gender, residency program, geographic location, and current position or trainee status; 2) mentorship in various stages of training; 3 ) family planning, fertility, and attitudes toward fertility treatments and breastfeeding; and 4) experiences with workplace culture and harassment. Most answers were stratified by gender.

\section{Statistical Analysis}

Data analysis was conducted in Microsoft Excel (version 2002, Microsoft Corp.). Continuous variables were presented as means and were tested using a 2-sided t-test. When comparing scores within an individual's responses, a paired t-test was used. Statistical significance was set at a $p$ value $<0.05$. The comparative results reported are significant if followed by a $\mathrm{p}$ value.

\section{Results}

According to the most recent available data, women currently compose $19.07 \%$ of total neurosurgery residents (288 female residents in a total of 1510 neurosurgery residents at 115 Accreditation Council for Graduate Medical Education (ACGME)-accredited Doctor of Medicine programs). The average number of female residents per program is 2.74 (range 0-9), with 14 total residents in most programs.

\section{Demographics}

A total of 115 respondents (64 men, 51 women; age range 25-67 years) had trained at 49 different US residencies and included 26 attendings, 5 fellows, and 84 residents. The average expected age at graduation for current residents is 34.3 years for men and 33.5 years for women. Geographic and demographic information was not significantly different between male and female respondents and is summarized in Table 1 and Fig. 1.

\section{Mentorship}

In medical school and residency, the overwhelming majority of women (90\% and $86 \%$, respectively) and men (86\% and $83 \%$, respectively) had a neurosurgery mentor. The percentage of medical students who had a female mentor was not significantly different between men $(5.5 \%)$ and women (13.3\%), but two-thirds of respondents with female mentors were women. There was a significantly greater rate of female residents with a female mentor $(16.7 \%)$ versus male residents with a female mentor $(3.8 \%$; $\mathrm{p}<0.05)$. Prior to residency, $80 \%$ of women and $88 \%$ of men reported concerns about residency. For $12 \%$ of women, the major concern with residency was the ability to thrive in a field with a majority of men, which was not a reported concern among men.

\section{Family Planning}

Regarding external pressures on starting a family, respondents used a scale of 1 (significant pressure to start a family) to 5 (significant pressure against starting a family) to rate pressure from programs or colleagues and from partners and family. About half of all surveyed women 
TABLE 1. Summary of demographics for survey respondents

\begin{tabular}{cccc}
\hline & & No. $(\%)$ & \\
\cline { 2 - 4 } Factor & Female & Male & Total \\
\hline Total no. of respondents & $51(44)$ & $64(56)$ & $115(100)$ \\
\hline Stage of training & & & \\
\hline Attending & $10(38)$ & $16(62)$ & $26(23)$ \\
\hline Fellow & $2(40)$ & $3(60)$ & $5(4)$ \\
\hline Resident & $39(46)$ & $45(54)$ & $84(73)$ \\
\hline Chief resident & $7(44)$ & $9(56)$ & $16(19)$ \\
\hline Senior resident & $16(43)$ & $21(57)$ & $36(43)$ \\
\hline Junior resident & $17(50)$ & $17(50)$ & $32(38)$ \\
\hline Age at residency graduation (yrs) & 33.5 & 34.3 & \\
\hline Geographic region* & 51 & 61 & 112 \\
\hline Northeast & $9(47)$ & $10(53)$ & $19(17)$ \\
\hline Southeast & $9(41)$ & $13(59)$ & $22(20)$ \\
\hline Mid-Atlantic & $4(31)$ & $9(69)$ & $13(12)$ \\
\hline Southwest & $3(27)$ & $7(70)$ & $10(9)$ \\
\hline Mid-West & $14(52)$ & $13(48)$ & $27(24)$ \\
\hline Northwest & $1(25)$ & $3(75)$ & $4(4)$ \\
\hline West & $11(65)$ & $6(35)$ & $17(15)$ \\
\hline Marital status & & & \\
\hline Married & $33 / 51(65)$ & $50 / 64(78)$ & $83 / 115(72)$ \\
\hline w/ children & $11 / 51(22)$ & $23 / 64(36)$ & $34 / 115(30)$ \\
\hline
\end{tabular}

* Of the 112 respondents trained in the United States.

felt at least some pressure from programs or colleagues against starting a family, compared with only $6 \%$ of male respondents who responded similarly (90\% of men reported no pressure from programs or colleagues, whereas $3 \%$ of men reported facing pressure to start a family; not a single woman reported this latter finding). All respondents who faced significant pressure against starting a family from programs or colleagues were women $(n=10)$.

Men experienced significantly greater pressure from families and partners to start a family (average score 2.5) compared to no pressure from programs or colleagues to start a family (average score $3.0 ; \mathrm{p}<0.001$; Fig. 2). Women experienced significantly greater pressure from families and partners to start a family (average score 2.7) compared to the pressure they felt from programs or colleagues against starting a family (average score 3.7; $\mathrm{p}<$ 0.001 ). When women's perceptions of external pressures on starting a family were compared with those of men, women perceived significantly greater pressure against starting a family from programs or colleagues than men
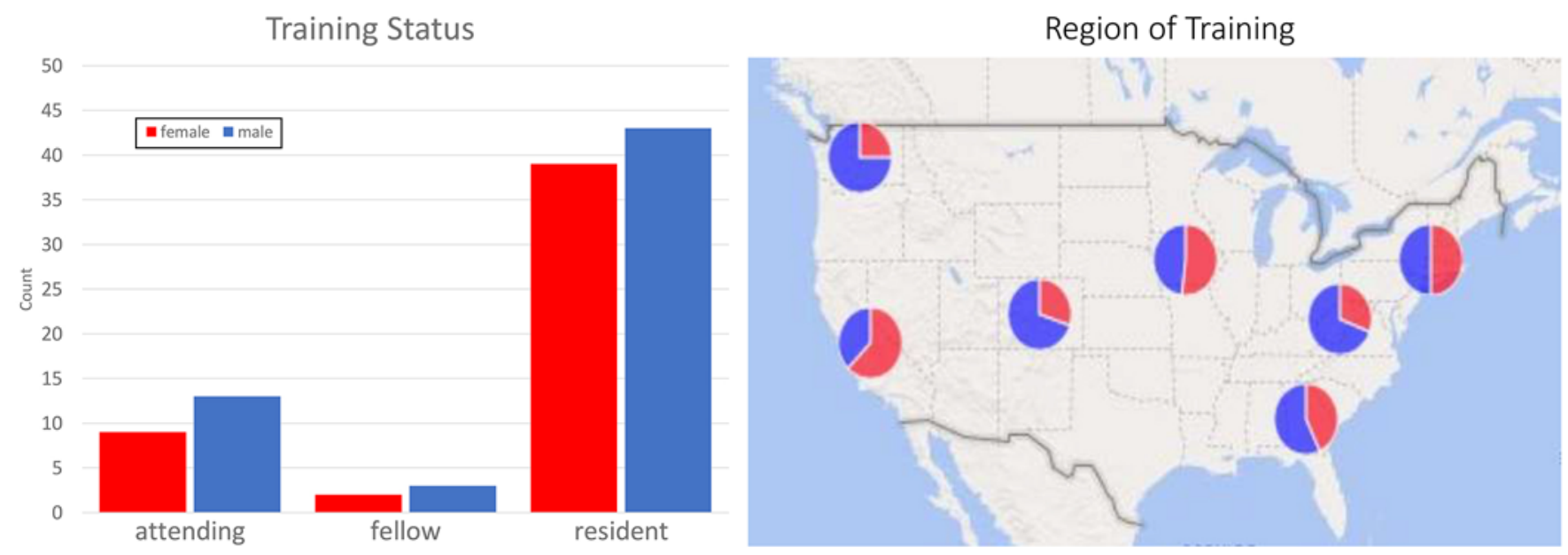

FIG. 1. Male and female respondents by training status and region of training. 


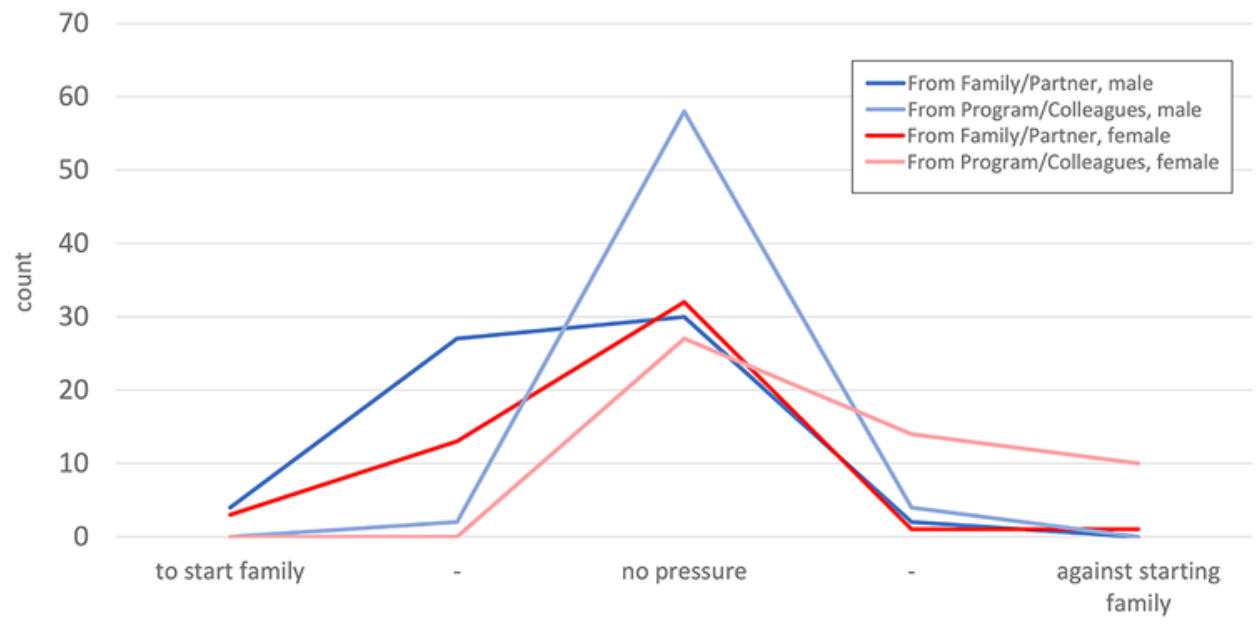

FIG. 2. Perceived pressure for or against starting a family among male and female respondents from their family/partner and their program/colleagues. Notably, male respondents perceived no pressure from their program and colleagues either for or against starting a family. Female respondents reported more pressure to start a family from their family/partner and more pressure against starting a family from their program/colleagues.

did $(p<0.001)$. Both men and women reported a similar amount of increased pressure from family and partners to start a family. More men (78.1\%) than women (66.0\%) were married.

Regarding personal preferences for childbearing, $42.2 \%$ of men wanted children prior to residency; however, only $10.9 \%$ had children prior to residency $(\mathrm{p}<0.001)$. For women, $34.0 \%$ wanted children prior to residency, whereas only $2.0 \%$ had children prior to residency (p < 0.001 ). There was not a significant difference between the percentage of men who wanted to have children during residency $(56.3 \%)$ and the percentage who had children during residency (44.1\%). However, there continued to be a significant difference between the percentage of women who wanted to have children during residency (48.0\%) and the percentage who had children during residency $(32.7 \%$; $\mathrm{p}<0.05)$.

For neurosurgeons who had a child during residency, significantly more women than men had a child only in a nonclinical year $(56.3 \%$ vs $19.0 \%$, respectively; $p<0.05)$. The average total number of children conceived was 1.9 for men and 1.5 for women. Among physicians with children, a female physician's average age at the birth of the first and second child was 31 and 34 years, respectively, compared to 29 and 31 years for males (Table 2).

Forty-two women respondents reported a strong desire to breastfeed whether or not they had had children, and on a scale of 1 (ambivalent) to 5 (very strongly in favor) women rated the importance of breastfeeding for them as 4.4 on average. This translated to a breastfeeding rate per child of $100 \%$ prior to residency, $94 \%$ during residency, and $100 \%$ after residency. Notably, one respondent reported a precipitous drop in the ability to produce breastmilk upon returning to work after maternity leave. On average, women during and after residency breastfed their child for 8 and 5.5 months, respectively.

Of the respondents who had tried to conceive, $25 \%$ of men and $39 \%$ of women reported difficulty conceiving.
The major perceived difficulty with childbearing/childrearing for men was stress, whereas women reported the physical challenges of pregnancy itself (including workplace teratogens and morning sickness). Of the women who were pregnant, failed births peaked for women neurosurgeons during residency at a rate of 0.33 compared to 0.00 before residency and 0.25 after residency (Fig. 3). For those respondents who reported difficulty conceiving, the average number of months of attempted conception was 37.5 for men and 25 for women. Of note, there were both men and women respondents who reported having ongoing difficulties with conception at the time of the study. The most common solution to conception difficulties for men was in vitro fertilization and chemical induction agents for women.

\section{Workplace Bias}

Women $(80.4 \%)$ reported discriminatory behavior in the workplace significantly more than men $(35.9 \%$; $\mathrm{p}<$ 0.001). Women experienced significantly higher rates of discrimination based on their gender $(95.1 \%)$ than men $(8.7 \% ; p<0.001)$. Both men and women experienced high rates of discrimination regarding their qualifications or training level (52.2\% and 46.3\%, respectively; Fig. 4). On multiple choice responses, the male senior residents/ attendings cohort was the most common source of this behavior, reported by $76.2 \%$ of men and $75.6 \%$ of women. For male respondents, this cohort was significantly more likely ( $\mathrm{p}<0.001$ ) to perpetrate macro-/microaggressions than all other groups provided, except for female nurses/ midlevel providers. For female respondents, this cohort was significantly more likely $(p<0.001$ ) to perpetrate macro-/microaggressions than all other groups provided, except for male patients. Additionally, male patients were significantly more likely to discriminate against women than men $(\mathrm{p}>0.001)$. 
TABLE 2. Family life of male and female neurosurgeons before, during, and after residency

\begin{tabular}{|c|c|c|c|}
\hline Factor & Before Residency & During Residency & After Residency \\
\hline \multicolumn{4}{|l|}{ Pregnancy complications } \\
\hline Training status ( $\mathrm{F}$ respondents) & 51 & 51 & 10 \\
\hline Failed birth rate per 100 (F respondents) & 0 & 19 & 4 \\
\hline Difficulty conceiving ( $\mathrm{F} \& \mathrm{M}$ respondents) & \multicolumn{3}{|c|}{ F: $9 / 23$ (39\%); M: 4/16 (25\%) } \\
\hline \multicolumn{4}{|l|}{ Breastfeeding (F respondents) } \\
\hline Strong desire to breastfeed & \multicolumn{3}{|c|}{$42(82 \%)$} \\
\hline Women who breastfed & 1 & 15 & 3 \\
\hline Breastfeeding rate per child & $100 \%$ & $94 \%$ & $100 \%$ \\
\hline Childbearing (F \& M respondents) & F: 51; M: 64 & F: 51; M: 64 & F: 10; M: 16 \\
\hline 0 children & F: 49; M: 54 & $\mathrm{~F}: 34 ; \mathrm{M}: 34$ & $\mathrm{~F}: 6 ; \mathrm{M}: 2$ \\
\hline 1 child & $\mathrm{F}: 1 ; \mathrm{M}: 7$ & F: 12; M: 22 & F: $3 ; \mathrm{M}: 1$ \\
\hline$>1$ child & $\mathrm{F}: 0 ; \mathrm{M}: 0$ & $\mathrm{~F}: 4 ; \mathrm{M}: 5$ & $\mathrm{~F}: 0 ; \mathrm{M}: 10$ \\
\hline \multicolumn{4}{|l|}{ Parenting (F \& M respondents) } \\
\hline Average age at 1st child (yrs) & \multicolumn{3}{|c|}{ F: 31.3; M: 29.3} \\
\hline Average no. of children & \multicolumn{3}{|c|}{$\mathrm{F}: 1.5 ; \mathrm{M}: 1.9$} \\
\hline Age btwn 1st \& last child (yrs) & \multicolumn{3}{|c|}{$\mathrm{F}: 3.2 ; \mathrm{M}: 4.1$} \\
\hline Child only during nonclinical year* & \multicolumn{3}{|c|}{ F: $56 \% ; M: 19.00 \%$} \\
\hline \multicolumn{4}{|l|}{ Favorable views on IVF (F \& M respondents) } \\
\hline Resident/fellow & \multicolumn{3}{|c|}{ F: 30 (73\%); M: 30 (63\%) } \\
\hline Attending & \multicolumn{3}{|c|}{ F: $3(30 \%) ; M: 1(6 \%)$} \\
\hline
\end{tabular}

\section{Discussion}

In 2011, Zhuge et al. ${ }^{9}$ posited that three major constraints hinder women's academic and leadership advancements in surgical specialties: 1) lack of effective mentors, 2) tradi- tional gender roles, and 3) manifestations of sexism in the medical environment. While the number of women entering neurosurgery has improved in the past few decades, our profession can still provide better support to propel the advancement of women in neurosurgical leadership and

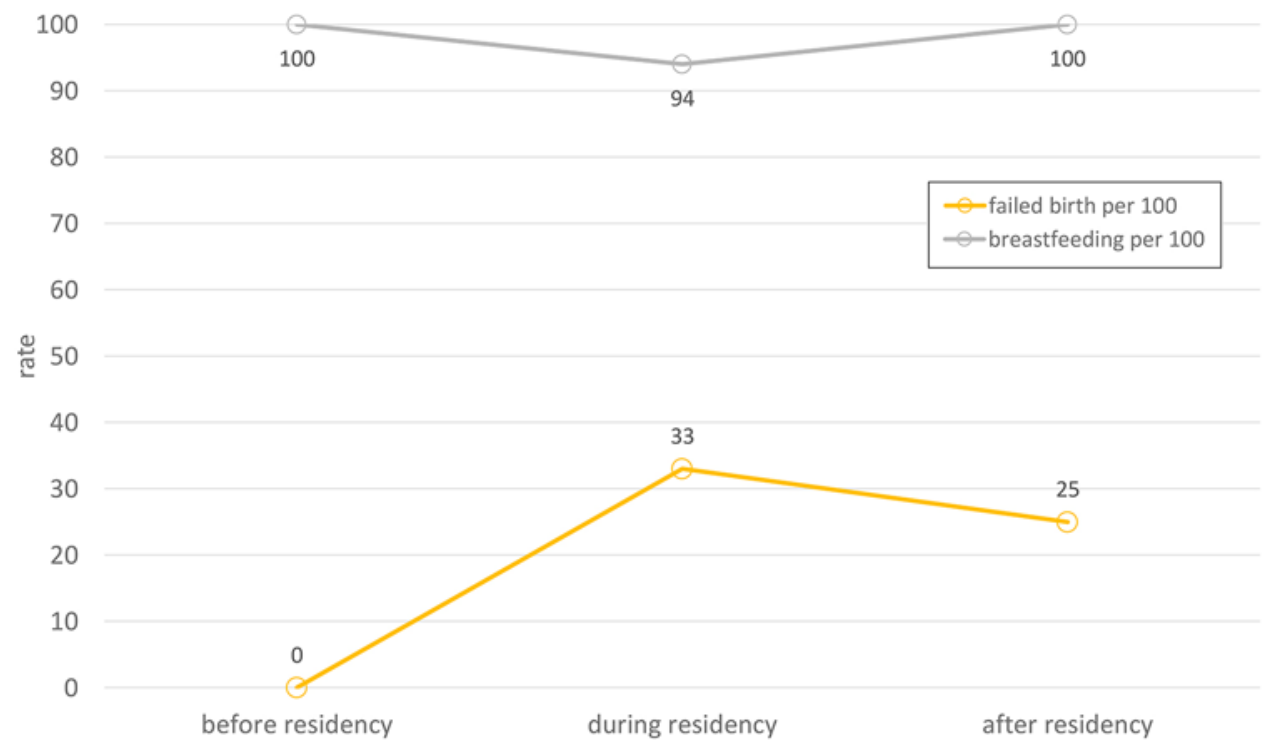

FIG. 3. Graph of infertility rate and breastfeeding-per-child percentage among women respondents for the three stages of training before, during, and after residency. An isolated rise in infertility rate and decrease in breastfeeding percentage was observed during residency compared to before and after residency, though neither was statistically significant. On average, during residency, breastfeeding duration was 8 months compared to 5 months for women who breastfed their child after residency. 


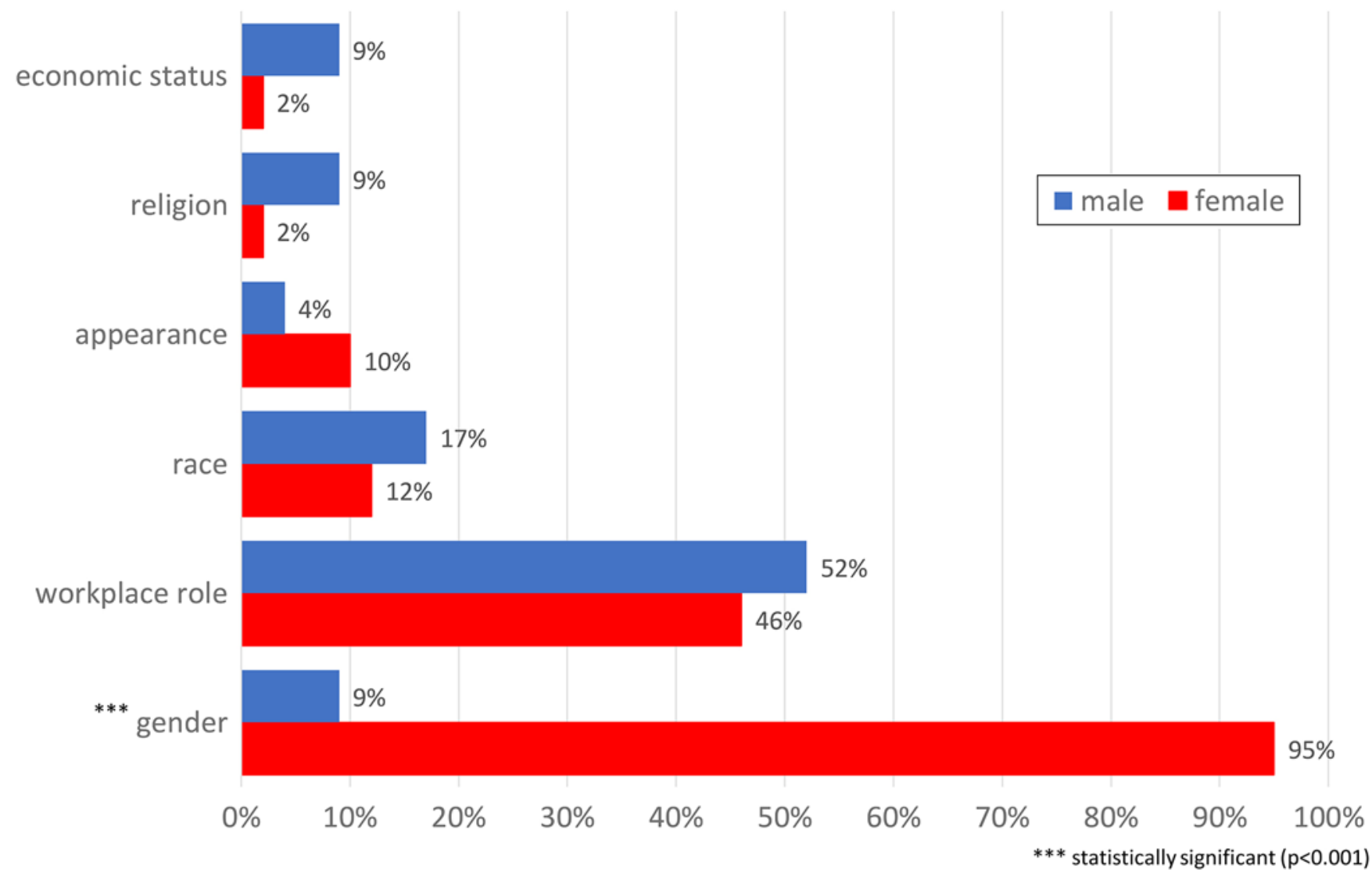

FIG. 4. Target characteristic of macro- and microaggressions as reported by percentages of male and female respondents. Each reported incidence of discrimination was categorized by characteristic (gender, workplace role, race, appearance, religion, and economic status). Female respondents reported statistically significantly more discrimination targeting their gender than male colleagues.

academia. The results of this survey may help to illustrate why there are still higher attrition rates for women than men in neurosurgery: a lack of diverse mentors for women neurosurgery trainees, diametrically opposed expectations and obligations of women at work and at home, and continued high rates of persistent workplace gender bias.

Another source of increased female attrition may be the larger disconnect between female neurosurgery applicants' expectations about the effects of neurosurgery on their personal life and career advancement compared to the actual experiences of women neurosurgeons in training and practice. This disparity between expectation and experience seems to be less pronounced for male applicants, including their ability to have children, if desired, during training. A recent survey of female neurosurgery applicants showed that applicants give the most weight to general, non-gender-specific considerations when evaluating programs (i.e., resident camaraderie and clinical experiences). They also showed that gender-specific considerations like the gender diversity of faculty and maternity leave policies ranked among the lowest in applicant considerations of programs. ${ }^{10}$ However, the results of our study would suggest that these gender-specific considerations seem to play a significant role in the experiences of current women neurosurgeons in training and practice.
This discrepancy may indicate that priorities for choosing a training program often remain focused around traditional academic and surgical metrics for both men and women. While "fit" is also described as an important selection criterion for all applicants, ${ }^{11}$ gender-specific factors are rarely highlighted as a component of training program culture and fit, perhaps because of the small number of women neurosurgeons in the field to share their experiences. In this study, we highlight some of these considerations to allow women applicants to understand the current landscape as well as the realities of where the field can improve.

\section{Mentorship}

Mentorship is a key factor for both men and women who successfully matriculated into neurosurgery. In medical school, male and female students were equally likely to have a female mentor. However, in residency, mentorship was significantly more likely to track along gender lines. Women more actively seeking female mentors during training may reflect the most common concerns of women regarding pursuing a career in neurosurgery: succeeding in a professional field with a majority of men.

The demand for female mentors by larger numbers of female trainees may create disproportionate mentorship 
responsibilities ${ }^{9}$ for the comparatively scarce women neurosurgical faculty. As Bennett et al. ${ }^{3}$ suggested, these significant efforts of senior female surgeons should be recognized and rewarded to improve women's mentorship and advancement in neurosurgery. This is a "minority tax" that can only be alleviated as more women advance in the field.

\section{Family Planning}

Significantly different challenges face women trying to balance pregnancy and motherhood with surgical training. Compared to men, who primarily reported stress-related challenges with family planning, women have additional biological and sometimes medical challenges they must contend with before, during, and after pregnancy. While both male and female neurosurgeons reported high rates of difficulty conceiving, the notably higher rate experienced by women and the increased rate of failed births that peaked during residency are consistent with data regarding pregnancy-related complications in female residents and night shift workers. More specifically, studies have shown that as few as two night shifts per week during weeks 3 through 21 of pregnancy increase the risk of miscarriage in a dose-dependent fashion ${ }^{12}$ and that longer operating hours and more than 6 nights on call per month are associated with elevated obstetrical complications in pregnant residents compared to those in an age-matched cohort. ${ }^{13}$ These inherent risks of call may explain why significantly more women respondents with successful pregnancies waited to have children during a nonclinical/ research year, compared to men. This delay may have contributed to the older age of women compared to men at the time of their first child's birth and the lower average number of children for women compared to men, which is consistent with other studies. ${ }^{14}$

During training, women neurosurgeons' significantly lower rates of childbearing compared to their desire was potentially attributable not only to risks to the mother and child associated with childbearing as a surgery resident, but also to the significantly increased perceived opposition by programs/colleagues to have children. Furthermore, consistent with other studies, ${ }^{14}$ fewer women were married or in a civil union than men, making family planning more difficult. Pregnancy and family planning remain important issues to address for female neurosurgical trainees and practicing neurosurgeons, ${ }^{15}$ as these pervasive, often gender-specific, challenges can lead to decreased job satisfaction and increased attrition rates. ${ }^{16}$

\section{Workplace Bias}

A recent survey of Congress of Neurological Surgeons members demonstrated that while practice patterns between male and female neurosurgeons are similar, female neurosurgeons experience significantly lower career fulfillment than their male counterparts and higher rates of sexism and harassment, ${ }^{14}$ findings consistent with our workplace discrimination findings. This is concerning given the association between higher rates of workplace mistreatment and higher rates of burnout and suicidal thoughts. $^{17}$

Senior male neurosurgeons were the most common perpetrators against both male and female respondents, and female nurses/midlevel providers were also common perpetrators. As neurosurgery attendings are currently majority male and nurses are majority female, respondents have a higher probability of interacting with these two groups of people at work. Survey results regarding male attendings and female nurses may therefore simply represent this underlying demographic distribution.

Conversely, patient demographics are likely approximately evenly distributed between genders; therefore, the significant greater discrimination against female providers compared to that against male providers from male patients could represent a meaningful finding. The identification of patients as a common source of gender discrimination is consistent with findings in studies among general surgery residents. ${ }^{17}$ Providers armed with the knowledge of such discrepancies can advocate for female colleagues as appropriate when they witness such behavior from patients to ensure a safer working environment.

\section{Study Limitations}

Limitations of this study include the limited sample size and possible response bias. Though the respondents were geographically diverse and represented all levels of training, only $13.5 \%$ (39/288) of female neurosurgery residents and a smaller percentage of the overall cohort of men in neurosurgery responded to this survey. Respondents who had strongly positive or negative experiences with any of the topics evaluated in this survey may have been more likely to complete the survey.

\section{Diversity in Neurosurgery}

While the focus of this study is specifically on gender, it is important to recognize racial and ethnic disparities in the field as well. Increased diversity in the neurosurgical workforce can help strengthen the field to become more representative of the patients that it endeavors to serve. ${ }^{18}$

\section{Conclusions}

Given historical gender disparities in neurosurgery, it is important to continue to track statistics on the gender gap to determine the effects of policy changes and to identify ongoing areas of improvement. Based on these survey results, potential changes may include 1) limiting terms in academic medicine ${ }^{19}$ to increase upward mobility and leadership opportunities for women in neurosurgery, which will also help to support the mentoring needs of the increasing number of female trainees; 2) increasing open discussions of the potential social, physical, and mental effects of childbearing/childrearing on trainees in neurosurgery, with special attention to increased fertility difficulties in women in particular, so women neurosurgeons can realistically approach family planning; 3) acknowledging that trainees are delaying having children because of career advancement or training restrictions and may experience greater difficulties with family planning because of it; 4) implementing policies to mitigate perceived risks of childbearing and childrearing (including decreased exposure to workplace teratogens, allowances or physician support for the physical effects of childbearing on women, decreased night shift work or support for pregnancy during less clini- 
cally intense years, and increased availability of child care and safe milk pumping and storage areas for postpartum women); and 5) actively identifying and mitigating workplace gender bias from all levels (including colleagues, superiors, support staff, and patients/families) to create a more conducive learning and working environment for trainees and attendings. This study broadly demonstrated obstacles for both men and women neurosurgeons regarding family planning, fertility, and workplace culture. As gender roles at home and at work become less pronounced, we have an opportunity as a profession to promote an environment that enables both men and women neurosurgeons to succeed in their personal and professional lives.

\section{Acknowledgments}

We thank all respondents for generously sharing their personal experiences with us through their survey responses.

\section{References}

1. WINS White Paper Committee; Benzil DL, Abosch A, Germano I, et al. The future of neurosurgery: a white paper on the recruitment and retention of women in neurosurgery. $J$ Neurosurg. 2008;109(3):378-386.

2. Durham SR, Donaldson K, Grady MS, Benzil DL. Analysis of the 1990-2007 neurosurgery residency match: does applicant gender affect neurosurgery match outcome? J Neurosurg. 2018;129(2):282-289.

3. Bennett CL, Baker O, Rangel EL, Marsh RH. The gender gap in surgical residencies. JAMA Surg. 2020;155(9):893-894.

4. Lynch G, Nieto K, Puthenveettil S, et al. Attrition rates in neurosurgery residency: analysis of 1361 consecutive residents matched from 1990 to 1999. J Neurosurg. 2015;122(2): 240-249.

5. Renfrow JJ, Rodriguez A, Liu A, et al. Positive trends in neurosurgery enrollment and attrition: analysis of the 2000-2009 female neurosurgery resident cohort. J Neurosurg. 2016; 124(3):834-839.

6. Renfrow JJ, Rodriguez A, Wilson TA, et al. Tracking career paths of women in neurosurgery. Neurosurgery. 2018;82(4): $576-582$.

7. Odell T, Toor H, Takayanagi A, et al. Gender disparity in academic neurosurgery. Cureus. 2019;11(5):e4628.

8. Woodrow SI, Gilmer-Hill H, Rutka JT. The neurosurgical workforce in North America: a critical review of gender issues. Neurosurgery. 2006;59(4):749-758.

9. Zhuge Y, Kaufman J, Simeone DM, et al. Is there still a glass ceiling for women in academic surgery? Ann Surg. 2011; 253(4):637-643.
10. McNutt SE, Goss ML, Hallan DR, Bible JE. Factors in residency decision making for female neurosurgery applicants. World Neurosurg. 2020;140:e105-e111.

11. Marasa LH, Pittman TA. Factors neurosurgery candidates use when choosing a residency program. J Neurosurg. 2014; 120(1):167-172.

12. Begtrup LM, Specht IO, Hammer PEC, et al. Night work and miscarriage: a Danish nationwide register-based cohort study. Occup Environ Med. 2019;76(5):302-308.

13. Behbehani S, Tulandi T. Obstetrical complications in pregnant medical and surgical residents. J Obstet Gynaecol Can. 2015;37(1):25-31.

14. Gadjradj PS, Matawlie RHS, Voigt I, et al. Gender differences between male and female neurosurgeons: is there equality for all? World Neurosurg. 2020;136:348-356.

15. Abosch A, Rutka JT. Women in neurosurgery: inequality redux. J Neurosurg. 2018;129(2):277-281.

16. Rangel EL, Smink DS, Castillo-Angeles M, et al. Pregnancy and motherhood during surgical training. JAMA Surg. 2018; 153(7):644-652.

17. Hu YY, Ellis RJ, Hewitt DB, et al. Discrimination, abuse, harassment, and burnout in surgical residency training. $N$ Engl J Med. 2019;381(18):1741-1752.

18. Kim EE, Klein AL, Lartigue JW, et al. Diversity in neurosurgery. World Neurosurg. 2021;145:197-204.

19. Beeler WH, Mangurian C, Jagsi R. Unplugging the pipeline - a call for term limits in academic medicine. $N$ Engl J Med. 2019;381(16):1508-1511.

\section{Disclosures}

The authors report no conflict of interest concerning the materials or methods used in this study or the findings specified in this paper.

\section{Author Contributions}

Conception and design: Thum, Liau. Acquisition of data: Thum, Chang. Analysis and interpretation of data: Thum, Chang, Tata. Drafting the article: Thum, Chang, Tata. Critically revising the article: Thum, Tata, Liau. Reviewed submitted version of manuscript: all authors. Approved the final version of the manuscript on behalf of all authors: Thum. Statistical analysis: Thum, Chang. Administrative/technical/material support: Thum, Liau. Study supervision: Liau.

\section{Correspondence}

Jasmine A. Thum: University of California, Los Angeles, CA. jthum@mednet.ucla.edu. 